\title{
Stigmatizing Role of Schizotypical Behavior on Social Anxiety during Pandemicamong Emerging Adults
}

\section{Sudha Rathore*, Mansi Chaudhary, Anchal Dwivedi}

Department of Psychology, IIS (Deemed to be University), Mansarovar, Jaipur (302020) Rajasthan, India

Study Area:Jaipur, India

Coordinates: $11^{\circ} 1^{\prime} 6^{\prime \prime} \mathrm{N} ; 76^{\circ} 58^{\prime} 21$ "E

Key words: Disorganized life, Anhedonia

\section{Abstract}

A sample of 282 people, comprising of 156 males and 126 females aged between 18-21 years of age were taken. The data collection tool used was Social Anxiety Questionnaire and O-Life Questionnaire was used. Findings revealed that there is a significant positive correlation between dimensions of Schizotyical behaviour- unusual experiences, Introverted Anhedonia, Cognitive Disorganization with Social Anxiety. Regression analysis revealed that the Predictor variable of the study viz., Unusual Experience (UE), Cognitive Disorganization (CD), Introverted Anhedonia (IA), and Impulsive Nonconformity contributed $12.2 \%$ variance in predicting social anxiety. The study will aid in predicting and controlling social anxiety considering these behavioral patterns which can further add up to the knowledge of beneficial treatments.

and further can work as a predictor to develop many other mental health issues. Seeing things that are not present, having disorganized thoughts, and social disinterest, can both be the cause or outcome of Social Anxiety, which is nothing but parts of Schizotyoical Behavioral patterns.

Schizotypy, on the other hand, includes experiences of cognition, and perceptual deformity which lead to many behavioural effect and mental well being. Schizotypal is made up of a series of several signs and symptoms reflecting cognitive, perceptual, social, interpersonal, and behavioural dysfunction. People suffering from this may experience perceptual alternations, such as hearing someone mumbling their name or sensing that a spirit is present when it's not. They may have unusual mannerisms, an individual with this disorder may wear ill-fitting clothing or bizarre clothing combinations that are evidently visible (winter boots with shorts) and may be unable to participate in the normal give-and-take or regular form of conversation, they often interact with people only when there is no way of avoiding it otherwise they choose not to. It can also be understood as a multifactorial construct with four core dimensions-

-Unusual experiences: the symptoms of these criteria are characterized by unusual perceptual and other cognitive experiences, namely, hallucinations, magical or superstitious beliefs or weird interpretations of events around them.

-Cognitive Disorganization: a tendency for thoughts or cognitions to become tangential, derailed, or distorted. end. Constant reinforcement to avoid such situations makes the urge to stay to themselves, even more, stronger,

*Corresponding Author: sudharathorevansh@gmail.com 


\section{TECHNOSCIENCE ARTICLE}

-Introverted Anhedonia: this state is usually characterized by introverted, emotionally flat and social behaviour where one fails to feel happy or pleased by social interaction.

-Impulsive nonconformity: this is when a person fails to conform to norms, or rules of the society or living standards usually because of mood instability. (Kwapil et al., 2012).

To talk about how both these concepts can interact with each other and affect the overall well being of an individual, there are past studies that talk about the same. Various studies have talked about Schizotypical Behavior and social anxiety, which shows relation or potential effect on the anxiety of a person. Findings suggest that people with Schizotypal traits were associated with lower overall life satisfaction, had higher chances of depression and anxiety in general. Dysfunction, Impulsivity, and antisocial patterns of behaviour were higher in these people, they were also found to be higher in hypomanic states, and had an overall negative effect. They find it difficult to maintain social relationships, family bonds, even when their emotional and cognitive abilities are unaffected, they also end up not being able to form intimate relationships, and find it difficult to form an identity in comparison to others without the Schizotypical Behavior of their age. All this suggests how Schizotypical people tend to have a lower or negative evaluation of life which can further lead up to having social anxiety. Hence, the sense of not having to pull positive feelings and maintain relationships can significantly affect people in social situations, resulting in them having higher levels of social anxiety and wanting to avoid such incidents. (Kemp et al., 2018; Aghvinian et al., 2018; Abott et al., 2011; Lewandowski et al., 2006; Dinn et al., 2002; Moon et al., 1997).

Overall, studies and researches suggest significant relations in between Schizotypical traits and the presence of social anxiety within people, how these traits can predict social anxiety within people. This aspect of social anxiety can have a self stigmatizing effect on oneself and leads to isolation. Keeping the current scenario in perspective it has inculcated a sense of mental ill-health among Indian youth. And looking at the dearth of researches in the Indian context it is important to study these variables among Indian youth. Furthermore, having to find out interventions and potential solutions that can help people deal with these traits can also help in predicting and controlling social anxiety and in turn, makes the study intriguing and relevant.

Objective of this study was to:

- To investigate the relationship between Schizotypical Behavior and Social Anxiety.

- To study the interactive effect of Schizotypical dimensions on Social Anxiety.

\section{Methodology:}

Based on the objectives of the study, the researchers have framed the following alternate

$\mathrm{H}_{1}$ : There will be a significant relationship between Schizotypical
Ambient Science, 2022: Vol. 09(1); 17-20 DOI:10.21276/ambi.2022.09.1.ta01

Behavior and Social Anxiety among emerging adults.

$\mathrm{H} 2$ : The independent variable of the study, Schizotypical Behavior will contributevariance in predicting the dependent variable, which is Social Anxiety among emerging adults.

Sample was $282(\mathrm{~N}=\mathbf{2 8 2})$, a mixed sample of males and females aged between 18-21 years. The inclusion criteria were the ability to comprehend vocabulary, and the exclusion criteria included no physical or mental deformity.

The sample was collected on the basis of the inclusion and exclusion criteria as mentioned above. As the consent was taken from the participants, the conduction of the relevant tools was done through a Google form, i.e. online mode. The statistical analysis was conducted on the collected data and the trends were reported accordingly.

\section{Data collections:}

1. Social Anxiety Scale (SAQ-30): Caballo (2010), the SAQA3o contains 30 items conforming to a social phobia/anxiety structure. The responses range from not at all or very slightly to extremely high, which leads to score ranging from 1 to 5 respectively. Possible scores can range from 30 to 150 for each individual. For the psychometric properties, the internal consistency coefficient was (0.92) and convergent validity was found significant.

2. O-LIFE (Oxford Liverpool Inventory of Feelings and Emotions) The O-LIFE questionnaire was made by Mason et al. (1995) to measure schizotypal traits. The questionnaire contains 43 items with four sub-scales: unusual experiences (aberrant beliefs and perceptions), cognitive disorganization, introvertive anhedonia (social disinterest), and impulsive non-conformity ( going against norms) and the type of responses remain Yes or No. As for psychometric properties, high internal consistency and test-retest reliability was found.

Social Anxiety can be predicted from the presence of Schizotypical Behaviour was the basis of our research protocol. Product Moment Correlation and Regression Analysis were used for data analysis.

\section{Results:}

Table-1 shows correlation analysis of independent variables viz. Introverted Anhedonia, Cognitive Disorganization, Impulsive Non-conformity, and Unusual experiences with Social Anxiety. It reveals a significant positive correlation with unusual experiences, cognitive disorganization, and Introverted Anhedonia.

Table-2 shows the result of separate multivariate analysis used to find out whether the independent variable of the study viz. Unusual Experience (UE), Cognitive Disorganization (CD), Introverted Anhedonia (IA), Impulsive Non-conformity predict Social Anxiety. The independent variables of the study together contribute $12.2 \%$ variance in predicting social anxiety which is found to be significant at 0.01 level $(\mathrm{F}=10.796)$. 
Table 1: Bivariate Correlational Analysis showing correlation between independent variables of Schizotyoical dimensions and dependent variable Social Anxiety $(\mathrm{N}=282)$.

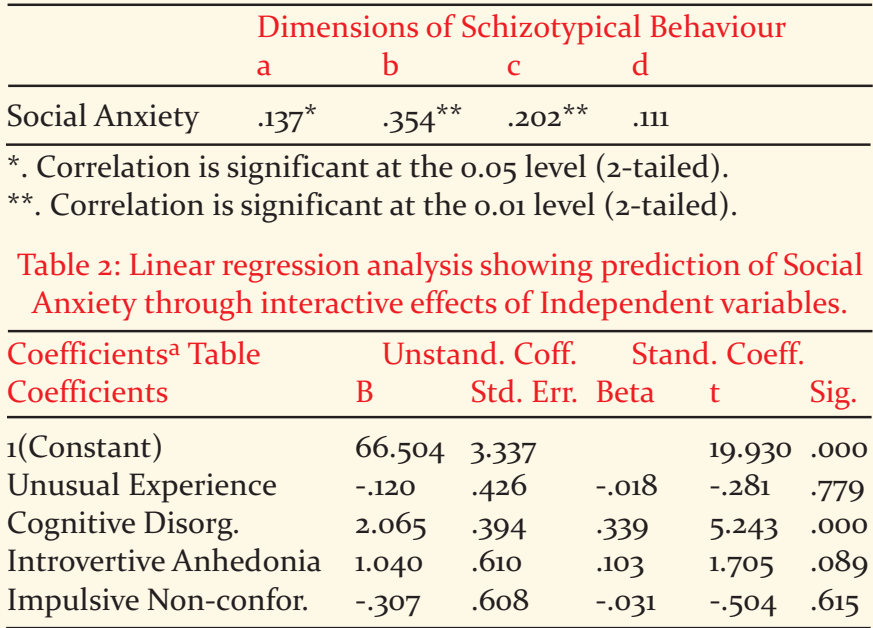

aDependent Variable: Social Anxiety

(NOTE: Fit for Model R2 =.135 Adjusted R2=.122; F=10.796, p<0.0o1)

\section{Discussion:}

Social Anxiety is not a novel concept that needs to be defined, but it constantly needs to be considered when we talk about the overall well being of human strata. People with Social Anxiety find it difficult to live a life where there is peace, and there may be many underlying causes of Social anxiety but various concepts go unnoticed, Schizotypical Behaviour is one of the same. Schizotypical Behaviour is characterized by disorganization of thoughts, and unreal cognition, where a person may see social surroundings as dangerous or punitive. The purpose of this study was to investigate the relationship between Schizotypical Behaviour and Social Anxiety among emerging adults. The independent variable of the study was unusual experiences, cognitive disorganization, introverted anhedonia, and impulsive non-conformity within Schizotypical Behaviour dimensions, and the dependent variable was Social Anxiety. The interactive effect of the independent variable on the dependent variable of the study was also investigated. A sample of 282 people of both males and females were taken for the conduction of this investigation.

The first hypothesis mentioned that there would be a significant relationship between Schizotypical Behaviour and Social Anxiety, was accepted. Results as per Table 1 indicated that dimensions unusual experiences have a significant positive relationship $(\mathrm{r}=0.137, \mathrm{p}<0.05)$, Cognitive Disorganization has significant positive correlation $(\mathrm{r}=.354, \mathrm{p}<0.01)$, Introverted Anhedonia also has a significant positive correlation $(r=.202, p<0.01)$ with dependent variable Social Anxiety. The second hypothesis mentioned that the independent variables of the study will contribute to variance in Social Anxiety among adults. The analysis of results in Table 2 clearly indicated that the independent variables of the study, viz Introverted Anhedonia, Cognitive Disorganization, Impulsive Non- conformity, and Unusual experiences together contributed $12.2 \%$ variance towards predicting Social Anxiety, whereas cognitive disorganization can alone predict the same, found to be significant at 0.01 level $(\mathrm{F}=10.796)$

These findings clearly suggest that people who have a higher level of schizotypical behavioural patterns within unusual experiences, cognitive disorganization, and introverted anhedonia reported higher on Social Anxiety. This suggests that Schizotypy affects the level of anxiety within an individual and can worsen the case if persistent without treatment. To give it a theoretical basis, Elis (https://study.com/academy/lesson/albert-ellis-theorylesson-quiz.html) explained the complications of irrational thoughts and cognitions that can lead to disturbed and distorted behavioural patterns. It is their beliefs, and values about themselves or the world that motivates them to think in a certain way and act accordingly. To relate with Schizotypal behaviour, it is those irrational beliefs, and cognition of an individual which reinforce them to act or behave that may be maladaptive, and further following this cycle, a person would start feeling deviated from himself or the outer world resulting in increasing social anxiety. The findings can also be supported by past studies as well, Premkumara, et al. (2018) found that people with higher level of interpersonal schizotypy were related to greater intensity of rejection sensitivity, which leads to difficulty in not only having positive feelings, but also in maintaining trust within a relationship, and processing pro social behaviour, which further elicits anxiety provoking episodes. Another researcher Brown et al. (2008) in his study reflects that there is a significant relationship between social anxiety and positive schizotypy, and further, the correlation was found with Social Anhedonia with Social Anxiety, and together both affected Schizotypy in different ways. Another study by Henry et al. (2008) mentioned that higher Schizotypy and behavioral pattern are related to lower social functioning of an individual thus further making them socially impaired. Both the studies can be used to indicate how Schizotypy affects an individual in a social context, and provide as a basis or mediator for increasing or predicting levels of Social anxiety.

\section{Implication:}

Within the current investigation, there are some implications that can be beneficial to get into our knowledge to have a better comprehension of how people deal with Social anxiety in terms of Schizotypy and how improvements can beattained.

- This research implicates that there are clear potential benefits to delivering the effective intervention in the population concern. However, there is limited evidence on the specific efficacy of available therapies for Schizotypy. Therefore we can incorporate counselling modules or develop strategies and interventions for tapping and 


\section{TECHNOSCIENCE ARTICLE}

mitigating social anxiety among emerging adults when considering Schizotyoical behaviour.

- Although, immense progress has been made to understand social anxiety, but still some topics remain undiscovered/unresolved. Thus, in future research studies, there is a significant scope of improvement to explore unresolved issues related to social anxiety in correlation to Schizotypy as the data of the same would be enhanced.

- Studies reflect how Schizotypy may start to develop during younger years of life, and thus the research provides a basis for future researchers to work on interventions that can be helpful in understanding patterns of Schizotypy at a young age, and how to move forward in order to eradicate the same, so to avoid higher chances of Social Anxiety later in life.

\section{References:}

Abbott, G.R., Do, M. \& Byrne, L.K. (2012): Diminished subjective well being in Schizotypy is more than just negative affect. Person. Indiv. Differ., 52(8):914-918.

Aghvinian, M. (2018): Social functioning impairment in schizotypy when social cognition and neurocognition are not impaired. Schizophrenia Res.: Cognit., 14:7-13.

Brown. L.H, Silvia. P.J, Germens. M.I, Lewandowski. K.E \& Kwapil. T.R. (2008): The relationship of social anxiety and social anhedonia to psychometrically identified schizotypy. J. Soc. Clin. Psycho., 27(2):127-149.

Caballo, V.E., Salazar, I.C., Irurtia, M.J., Arias, B., \& CISO-A Research Team. (2012): The multidimensional nature and multicultural validity of a new measure of social anxiety: The Social Anxiety Questionnaire for adults. Behav. Ther. 43(2):313-328.
Ambient Science, 2022: Vol. 09(1); 17-20 DOI:10.21276/ambi.2022.09.1.ta01

Dinn, W.M., Harris, C.L., Aycicegi, A., Greene, P., \& Andover, M.S. (2002): Positive and negative schizotypy in a student sample: neurocognitive and clinical correlates. Schizophrenia Res., 56(12):171-185.

Henry, D., Bailey, E. \& Rendell, G. (2008). Empathy, social functioning, and Schizotypy. Psych. Res., 160(1):15-22.

Kemp, K.C., Gross, G.M., Vidal, N.B. \& Kwapil, T.R. (2018): Association of positive, negative, and disorganized schizotypy dimensions with affevtive symptoms and experiences. $\underline{\text { Psych. }}$ Res., 270:1143-1149.

Lewandowski, K.E., Vidal, N.B., Gray, R.O., Clancy, C., Kepley, H.O. \& Kwapil, T.R. (2006): Anxiety and depression Symptoms in psychometrically identified schizotypy. Schizophrenia Res., $83(2-3): 225-235$.

Mason, O. \& Claridge, G. (2006): The oxford Liverpool Inventory of Feelings and Emotions (O LIFE). Schizophrenia Res., 82(23):203-211.

Moon, H.O., Yang, I.H., Lee, H.P., Kim, M.E. \& Ham, W. (1997): The preliminary study on the validation of schizotypal questionnaire. J. Korea. Neuropsych. Asso., 36(2):329-343.

Silvia, P.J, \& Kwapil, T.R (2010). Aberrant Asociality: How Individual Differences in Social Anhedonia Illuminate the Need to Belong. L. Personal., 79(6):1315-1332.

Premkumar. P, Onwumere. J, Betts. L, Kibowski. F, \& Kuipers. E. (2018). Schizotypal traits and their relation to rejection sensitivity in the general population: Their mediation by quality of life, agreeableness and neuroticism. Psych. Res., 267:201-209. 